\title{
Melatonin protects mouse oocyte from heat stress damage
}

\author{
Haiyan Du, Shouhong Wang, Weiwei Huang* \\ Reproductive Medicine Center, Department of Obstetrics and Gynecology, the Third Affiliated Hospital of Inner Mongolia \\ Medical University, Baotou, Inner Mongolia 014010, China
}

Received: December 22, 2019

Accepted: March 12, 2020

Online Published: April 22, 2020

DOI: $10.5430 /$ dcc.v6n3p13

URL: https://doi.org/10.5430/dcc.v6n3p13

\begin{abstract}
Objective: To explore the potential effect of melatonin on the in-vitro maturation of mouse oocytes under heat shock condition. Methods: This study used a heat shock model of mouse oocyte maturation. The oocytes were randomly divided into three groups: control group, heat shock group and heat shock + melatonin group, in order to evaluate the effect of $1 \times 10^{-9} \mathrm{~mol} / \mathrm{L}$ melatonin on the quality of oocytes after heat shock.

Results: In comparison with the control group, the maturation rate of mouse oocytes in heat shock group was significantly decreased [ $(33.00 \pm 0.07) \%$ vs. $(85.00 \pm 0.03) \%, p<.01]$, with abnormal spindle assembly, and the early apoptosis rate was significantly increased $[(59.7 \pm 4.5) \%$ vs. $(22.0 \pm 3.5) \%, p<.01]$. Compared with heat shock group, the maturation rate of oocytes was significantly increased in heat shock + melatonin group [(70.00 \pm 0.05$) \%$ vs. $(33.00 \pm 0.07) \%, p<.01]$, meanwhile, the spindle abnormality rate and the early apoptosis rate were significantly decreased accordingly [ $(37.3 \pm 6.1) \%$ vs. $(59.7 \pm$ $4.5) \%, p<.01]$. The expression level of heat shock protein 70 was significantly up-regulated in heat shock + melatonin group in comparison with other two groups $(p<.01)$.

Conclusions: By regulating the over-expression of heat shock protein 70, melatonin can improve the declined maturation rate of oocytes and the increased rates of spindle assembly abnormality and early apoptosis caused by heat shock.
\end{abstract}

Key Words: Heat shock, Oocyte, Melatonin, Heat shock protein 70

\section{INTRODUCTION}

Mammals have a capability to keep physiological function in the range from 35 to $39^{\circ} \mathrm{C}$. To keep body temperature constant is vital for living bodies to maintain normal physiological function. Under the condition of sustained high temperature, the animal body can respond to the severe environment by a series of physiological and pathological changes. If the external temperature is too high, the regulating ability of body will be suppressed. When the temperature reaches up to $41^{\circ} \mathrm{C}$, most of animals can't stand this type of stress, to the extent that the physiological function will be impaired. ${ }^{[1]}$ The reproductive system of mammals is more sensitive to temperature than any other parts of body. A large number of researches have shown that heat stress (HS) severely affects mammal spermatogenesis, oocyte maturation, early embryo development and fetal growth and development. HS can lead to oocyte dysmaturity by various approaches, probably affecting the synthesis of steroid hormones (such as LH) in granule cells; ${ }^{[2]} \mathrm{HS}$ can impair chromosomes and cytoskeleton of in-vitro maturated oocytes, and then decrease the embryo development. ${ }^{[3]}$ Other researches also show that HS

*Correspondence: Weiwei Huang; Email: nmgbgyyyy@ 163.com; Address: Reproductive Medicine Center, Department of Obstetrics and Gynecology, the Third Affiliated Hospital of Inner Mongolia Medical University, Baotou, Inner Mongolia 014010, China. 
can induce the apoptosis of porcine granule cells, accelerate follicular atresia, and significantly decrease the ovulation rate. ${ }^{[4]}$ With the suppression of apoptosis-related gene caspase, the adverse effect of HS on the developmental potentiality of bovine embryos is significantly decreased. ${ }^{[5]}$ In mouse oocytes, HS promotes the over-expression of reactive oxygen species (ROS), decreases the content of glutathione, and consequently results in the occurrence of oxidative stress responses. ${ }^{[6]}$ However, oxidative stress response, one of the most common factors impairing the normal physiological function of cells, can result in the destruction of intracellular structures and lead to cell death. Antioxidants can improve cell living environment and reduce the damage of HS to cells. Melatonin (N-acetyl-5-methoxytryptamine) is one of the most effective oxidants. Melatonin plays an important role in the maturation of oocytes, ovulation and the development of early embryos etc. The research results show that melatonin can improve the quality of bovine oocytes and then improve the embryo development, ${ }^{[7]}$ and it is probable to be a novel adjuvant drug for the treatment of infertility. ${ }^{[8]}$ Even though many researches have reported the promotion effect of melatonin on the in-vitro maturation of oocytes, the specific mechanism remains unclear yet. This research is designated to identify the potential relationship of HS with the maturation and the impairment of oocytes by establishing a HS model of oocytes. Furthermore, it is also intended to explore the functional mechanism of melatonin on promoting the maturation of oocytes under the condition of HS by adding melatonin in vitro.

\section{MATERIALS AND METHODS}

\subsection{Experimental animals}

8-week-old ICR mice were purchased from SPF Experimental Animal Breeding Center of Inner Mongolia University, raised under the temperature of 22 to $24^{\circ} \mathrm{C}$, the humidity of $40 \%-60 \%$, and the lighting condition (12 h light/dark cycle). These mice were fed with sterile pelletized feed, and had free access to food and water. All animal experiments involved in this research were performed in accordance with "Manipulative Technique for the Care and Use of Laboratory Animals" of Inner Mongolia Medical University.

\subsection{Research methods}

\subsubsection{The collection and the in-vitro maturation of oocytes}

8-week-old ICR female mice were given subcutaneous injection of $10 \mathrm{U}$ of pregnant mare serum gonadotropin (PMSG) respectively, and sacrificed by means of cervical dislocation after $48 \mathrm{~h}$. Mouse ovaries were taken out and punctured to collect cumulus-oocyte complexes. Glass needles with an appropriate diameter were used to repeatedly blow the complexes to remove cumulus cells. The remaining oocytes were cultivated in CZB culture medium (Sigma, USA) and placed into the incubator $\left(37^{\circ} \mathrm{C}, 5 \% \mathrm{CO}_{2}\right)$. On the time point of 2.5 $h$, it was required to calculate the rate of germinal vesicle breakdown (GVBD), i.e., GVBD rate $=$ (the number of cells in GVBD/the total number of cells) $\times 100 \%$; on the time point of $14 \mathrm{~h}$, the rate of first polar body extrusion (PBE) was calculated, that is, $\mathrm{PBE}$ rate $=($ the number of oocytes with $\mathrm{PBE} /$ the total number of cells) $\times 100 \%$.

\subsubsection{HS treatment and medication treatment}

The oocytes were randomly divided into three groups: control group (Ctrl group), heat shock group (HS group) and heat shock + melatonin group (HS + M group). The concentration of melatonin (Sigma, USA) was $1 \times 10^{-9} \mathrm{~mol} / \mathrm{L} .^{[9]} \mathrm{Cul}-$ ture droplets were kept overnight to attain equilibrium. The oocytes in the control group were placed into CZB culture medium containing $2.5 \mu \mathrm{mol} / \mathrm{L}$ of milrinone (Sigma, USA) to make oocytes keep in $\mathrm{GV}$ phase and cultivated under the condition of $37^{\circ} \mathrm{C}$ and $5 \% \mathrm{CO}_{2}$ for $10 \mathrm{~h}$; the oocytes in $\mathrm{HS}$ and $\mathrm{HS}+\mathrm{M}$ groups were placed into $\mathrm{CZB}$ culture medium containing $2.5 \mu \mathrm{mol} / \mathrm{L}$ of milrinone and cultivated in the incubator $\left(42^{\circ} \mathrm{C}, 5 \% \mathrm{CO}_{2}\right)$ for $10 \mathrm{~h}$ for $\mathrm{HS}$ treatment. Subsequently, 3 groups of oocytes were immersed into droplets to rinse out milrinone and then transferred to the incubator $\left(37^{\circ} \mathrm{C}, 5 \% \mathrm{CO}_{2}\right)$ for maturation culture.

\subsubsection{Fluorescence staining of spindles and chromosomes}

The oocytes were collected and then placed into Tyrode's solution to remove zona pellucida, subsequently fixed with $4 \%$ PFA for 30 min, permeated with $0.5 \%$ Triton X-100 for 1 $\mathrm{h}$ and blocked in $1 \% \mathrm{BSA}$ for $1 \mathrm{~h}$ at room temperature; incubated overnight in primary antibody anti- $\beta$-tubulin (Abcam, USA) under the ratio of $1: 2,000$ at $4{ }^{\circ} \mathrm{C}$, and then incubated for $1 \mathrm{~h}$ in a light-proof place in 1:1,000 secondary antibody FITC-Donkey Anti-Rabbit IgG (Jackson ImmunoRearch, USA) at room temperature. Later, these cells were re-stained with 1:50 PI (Sigma, USA) for $10 \mathrm{~min}$ at room temperature and mounted to be observed and imaged under the confocal microscope.

\subsubsection{The measurement of the early apoptosis of oocytes}

Apoptosis detection kits were purchased from Nanjing Jiancheng Bioengineering Institute and used in the detection according to the operating instruction. The ratio of binding buffer and Annexin-V-FITC was set as 10:1. After the cells were incubated in a light-proof place for $10 \mathrm{~min}$, they were observed, counted and photographed under the confocal laser scanning microscope. The early apoptosis of oocytes can be indicated by green fluorescence signals, i.e., apoptosis rate $=($ the number of oocytes with fluorescence signals/the total number of cells) $\times 100 \%$. 


\subsubsection{The measurement of heat shock protein 70 by use of western blot}

The collected oocytes were added by $13 \mu \mathrm{l}$ of lysis buffer (Bio-rad, USA) to achieve HS protein 70, which was then boiled for $5 \mathrm{~min}$ and loaded in $12 \%$ separating gel. The electrophoresis was performed under the constant voltage of $120 \mathrm{~V}$ for $150 \mathrm{~min}$. Wet transfer was used for transmembrane for $1 \mathrm{~h}$ under the current of $200 \mathrm{~mA}$. 5\% skim milk powders were used for blocking for $2 \mathrm{~h}$. The band position of the target protein was selected to overnight incubate first antibody AntiHsp70 (Abcam, USA) and 1:500 Anti- $\beta$-tubulin (Abcam, USA) respectively at $4^{\circ} \mathrm{C}$. Subsequently, $1: 2,000$ secondary antibody Perox-AffiniPure Dnk Anti-Rabbit IgG (Jackson ImmunoRearch, USA) was incubated for $1 \mathrm{~h}$ at $37^{\circ} \mathrm{C}$. After this process, HRP chemiluminescence ECI (Pierce, USA) was also incubated for measurement and imaging.

\subsection{Statistical analysis}

Each experiment in this study was made in triplicate at least, and SPSS 20.0 software was used to make an analysis of statistical data. The measurement data were represented by ( $\bar{X} \pm \mathrm{s}$ ), and the comparison of two groups was made by use of Student's $t$ test. The difference $(p<.05)$ was of statistical significance.

\section{Results}

\subsection{The effect of melatonin on promoting the matura- tion of HS-treated oocytes}

The statistical analysis was made by treating oocytes with HS and adding melatonin for the in-vitro maturation for $14 \mathrm{~h}$ after HS treatment. The results showed that, in comparison with the control group, GVBD rate and PBE rate were significantly decreased in HS group $(p<.01)$; after adding melatonin to the culture medium of HS-treated oocytes, in comparison with HS group, GVBD rate $(p<.05)$ and PBE rate $(p<.01)$ in $\mathrm{HS}+\mathrm{M}$ group were obviously increased, but still remarkably lower than the control group $(p<.01)$ (see Table 1, Figure 1).

Table 1. The effect of melatonin on the maturation of HS-treated oocytes $(\bar{X} \pm \mathrm{s})$

\begin{tabular}{llll}
\hline Group & N & GVBD Rate (\%) & PBE Rate (\%) \\
\hline Control Group & 129 & $96.00 \pm 0.04$ & $85.00 \pm 0.03$ \\
HS Group & 126 & $72.00 \pm 0.04^{*}$ & $33.00 \pm 0.07^{*}$ \\
HS + M Group & 115 & $80.00 \pm 0.03^{* \#}$ & $70.00 \pm 0.05^{*+}$ \\
\hline
\end{tabular}

Note. In comparison with the control group, ${ }^{*} p<.01$; in comparison with HS group, ${ }^{\#} p<.05,{ }^{+} p<.01$.
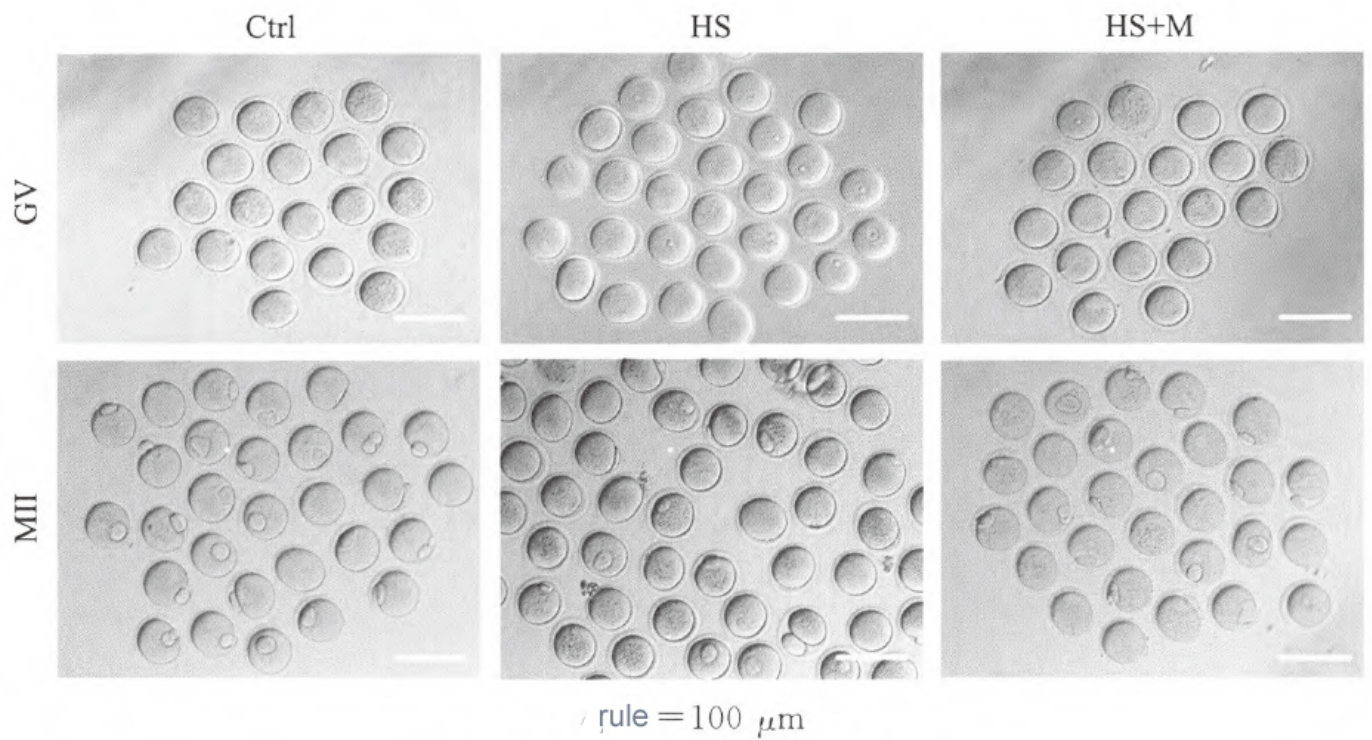

Figure 1. The maturity of oocytes in different treatment group

\subsection{The effect of melatonin on improving spindle abnor- mality of HS-treated oocytes}

In order to explore the mechanism of melatonin to improve the quality of oocytes, the microtubule condition of spindle was observed with the help of immunofluorescence staining. It was found that, in comparison with the control group, the abnormality of spindle assembly occurred in HS-treated oocytes, resulting in the incorrect arrangement of chromosomes to the equatorial plate (see Figure 2). Through counting the number of abnormal spindles, it was found that, in comparison with the control group, the rate of abnormal spindles in HS group was obviously increased $(p<.01)$; after adding melatonin, the abnormality of spindle assembly in $\mathrm{HS}+\mathrm{M}$ group was relieved to some extent in comparison 
with HS group $(p<.01)$, but didn't recover to the level in the control group $(p<.01)$ (see Table 2$)$.

Table 2. The effect of melatonin on the spindle abnormality rate of HS-treated oocytes $(\bar{X} \pm s)$

\begin{tabular}{lll}
\hline Group & N & Spindle Abnormality Rate (\%) \\
\hline Control Group & 129 & $11.8 \pm 2.5$ \\
HS Group & 126 & $55.9 \pm 2.7^{*}$ \\
HS + M Group & 115 & $36.0 \pm 7.4^{* \#}$ \\
\hline
\end{tabular}

Note. In comparison with the control group, ${ }^{*} p<.01$; in comparison with HS group, $\# p<.01$.

\subsection{The effect of melatonin on reducing the early apop- tosis of HS-treated oocytes}

The results concerning the early apoptosis of oocytes showed that the apoptosis rate of the oocytes in HS group was obviously higher than that in the control group $(p<.01)$; after adding melatonin, the apoptosis rate of HS-treated oocytes was decreased to some extent $(p<.01)$ (see Table 3, Figure 3).

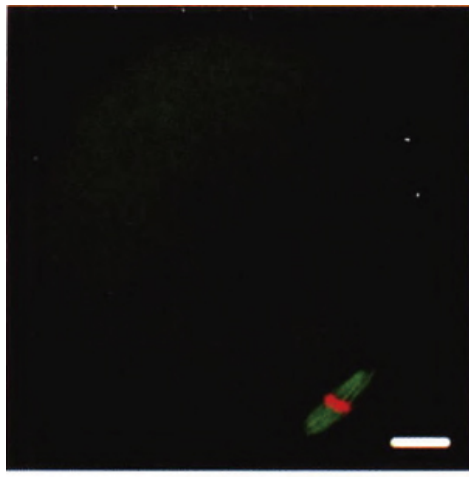

Ctrl

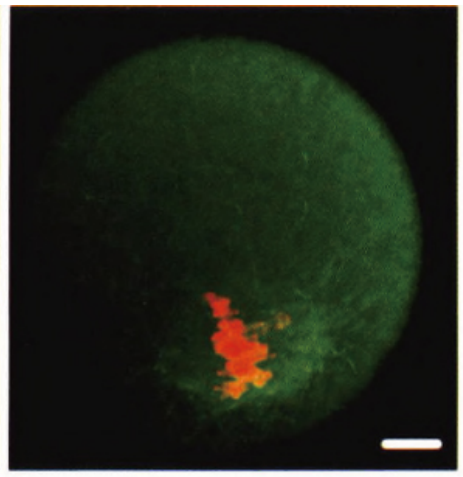

HS
Table 3. The effect of melatonin on the early apoptosis of HS-treated oocytes $(\bar{X} \pm \mathrm{s})$

\begin{tabular}{lll}
\hline Group & N & Apoptosis Rate (\%) \\
\hline Control Group & 129 & $22.0 \pm 3.5$ \\
HS Group & 126 & $59.7 \pm 4.5^{*}$ \\
HS + M Group & 115 & $37.3 \pm 6.1^{* \#}$ \\
\hline
\end{tabular}

Note. In comparison with the control group, ${ }^{*} p<.01$; in comparison with HS group, ${ }^{\#} p<.01$.

\subsection{The effect of melatonin on improving the quality of} oocytes by the over-expression of HSP70

The expression level of heat shock protein 70 (HSP70) was measured to explore the mechanism of melatonin to improve the quality of oocytes. The results showed that the expression level of HSP70 was up-regulated somewhat, but there was no significant difference in comparison with the control group ( $p>.05$ ); after adding melatonin, the expression level of HSP70 in HS + M group was obviously increased in comparison with the control group and HS group $(p<.01)$ (see Figure 4).

Figure 2. The condition of spindles in MII oocytes of different treatment groups (chromosomal complexes treated with immunofluorescence staining)

Red: Chromosomes; Green: Spindles; Scale $=20 \mu \mathrm{m}$

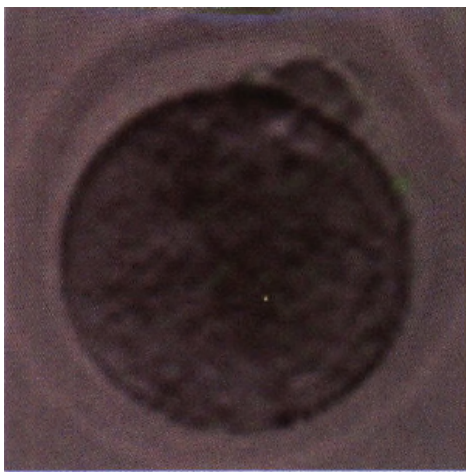

Ctrl

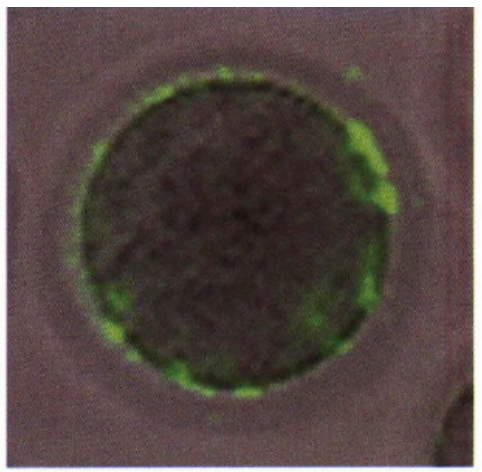

HS

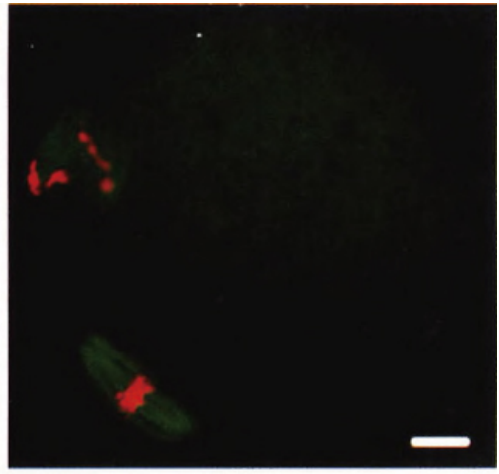

$\mathrm{HS}+\mathrm{M}$

Figure 3. The condition of the early apoptosis in MII occytes of different treatment groups

The signal of the early apoptosis was shown as green fluorescence on the oocyte membrane 

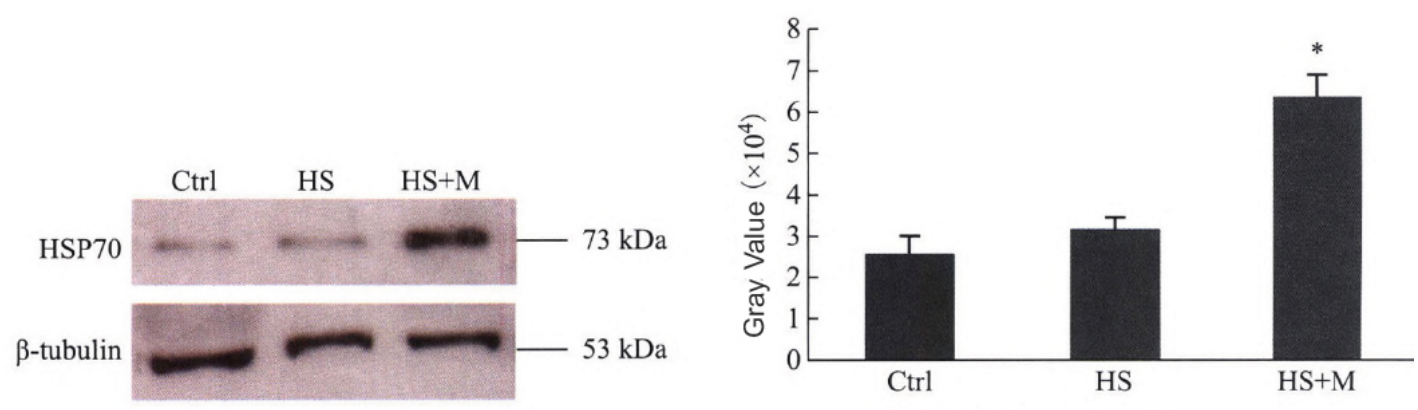

Figure 4. The expression of HSP70 in oocytes of different treatment groups In comparison with the other two groups, ${ }^{*} p<.01$

\section{Discussion}

Heat shock can result in oxidation damage to animal reproductive system, cause cell dysfunction and lead to cell apoptosis. For example, hyperthermia can remarkably induce the apoptosis of porcine ovarian follicles and granule cells. ${ }^{[10]}$ In $22 \mathrm{~h}$ after artificial insemination, if the porcine embryo is exposed to the temperature of $42^{\circ} \mathrm{C}$ for $9 \mathrm{~h}$, it will lead to the abnormal embryo development. ${ }^{[11]}$ The results of this research have shown that HS can significantly influence GVBD and PBE rates, which conforms to the research results from previous scholars. HS can affect the developmental potentiality of embryos by influencing the maturation of oocytes. ${ }^{[12]}$ By means of immunofluorescence staining, it has been found that HS has an effect on the assembly of microtubules during the maturation of oocytes, so that oocytes cannot extrude first polar bodies normally. The early apoptosis of oocytes has been also measured in this research, which indicates that HS can significantly increase the early apoptosis rate of oocytes, and the early apoptosis rate can be effectively reduced by adding melatonin, but it fails to recover to the level of the control group.

Melatonin is an effective antioxidant, altogether with its metabolites N1-acety-N2-methoxytryptamine and N1-acety5-methoxytryptamine, can directly eliminate reactive oxygens and stimulate the generation of antioxidant enzymes. Additionally, melatonin can reduce the generation of hydroxyl radicals by chelating transition metal ions. Therefore, by virtue of mechanism, melatonin can relieve oocyte dysmaturity caused by heat stress. Beyond all that, some researches have also shown that HSP70 is a type of multi-factor acting factor which can maintain intracellular homeostasis and suppress cell apoptosis. ${ }^{[13]}$ By regulating the levels of caspase-3 and cytochrome C, HSP 70 can prevent cells from apoptosis and play a role in the downstream of apoptosis regulatory network. High level of HSP70 can up-regulate SPKH1, BCL2, SOD1, CAT and CPX4 and down-regulate p53 in order to help oocytes to survive. ${ }^{[14-16]}$ Therefore, the measurement results in this research have shown that melatonin can improve the quality of oocytes by up-regulating the expression of HSP70 to protect oocytes.

\section{Conclusion}

HS can affect the maturation of oocytes under the condition of in-vitro culture. It may be caused by oxidative stress and the generation of disturbing hormones, which conversely lead to nuclear maturation and cell cycle arrest. Apart from that, by inhibiting the normal function of mitochondria, HS can lead to oocyte dysmaturity and even cause early apoptosis. Melatonin can significantly improve the maturation of oocytes by eliminating ROS and regulating the generation of steroid hormones. ${ }^{[17]}$ More essentially, melatonin can protect mitochondria from early apoptosis brought by stress damage. ${ }^{[18]}$ What's more, oocytes may have a selfprotection mechanism to enhance the generation of endogenous melatonin to change the expression of HSP70. All these mechanisms can help oocytes to cope with HS.

\section{CONFLICTS OF INTEREST DISCLOSURE}

The authors declare they have no conflicts of interest.

\section{REFERENCES}

[1] Cheshire WP Jr. Thermoregulatory disorders and illness related to heat and cold stress. Auton Neurosci. 2016; 196: 91-104. PMid:26794588. https://doi.org/10.1016/j. autneu. 2016. 01.001

Published by Sciedu Press
[2] Nteeba J, Sanz-Fernandez MV. Rhoads RP, et al. Heat Stress alters ovarian insulin-mediated phosphatidylinositol-3 kinase and steroidogenic signaling in gilt ovaries. Biol Reprod. 2015; 92: 148 PMid:25926439. https://doi.org/10.1095/biolreprod.114 .126714 
[3] Tremblay P, Gori A. Maguer JF, et al. Heterotrophy promotes the re-establishment of photosynthate translocation in a symbiotic coral after heat stress. Sci Rep. 2016; 6: 38112. PMid:27917888. https://doi.org/10.1038/srep38112

[4] Pavani KC, Baron E, Correia P, et al. Gene expression, oocyte nuclear maturation and developmental competence of bovine oocytes and embryos produced after in vivo and in vitro heat shock. Zygote. 2016; 24: 748-759. PMid:27019120. https ://doi.org/10.1017/S096 7199416000071

[5] Paula FF, Hansen PJ. Apoptosis js an adaptive response jn bovine preimplantation embryos that facilitates survival after heat shock. Biochem Biophys Res Commun. 2002; 295: 37-42. https://doi . org/10.1016/S0006-291X (02)00619-8

[6] Gharibzadeh Z, Riasi A, Ostadhosseini S, et al. Effects of heat shock during the early stage of oocyte maturation on the meiotic progression, subsequent embryonic development and gene expression in ovine. Zygote. 2015; 23: 573-582. PMid:24964001 https://doi.org/10.1017/S0967199414000203

[7] Wang F, Tian X, Zhang L, et al. Beneficial effect of resveratrol on bovine oocyte maturation and subsequent embryonic development after in vitro fertilization. Fertil Steril. 2014; 101: 577-586. PMid:24314921. https://doi.org/10.1016/j.fertnstert. 2 013.10 .041

[8] Wei-zhou W, Li-kun R, Yu-liang S, et al. Effect of melatonin supplementation on improving developmental potential of aging oocytes in IVF. Journal of Reproductive Medicine. 2017; 26: 470-476.

[9] Lan M, Han J, Pan MH, et al. Melatonin protects against defects induced by deoxynivalenol during mouse oocyte maturation. J Pineal Res. 2018; 65: e12477. PMid:29453798. https ://doi.org/10.1 $111 /$ jpi. 12477

[10] Sirotkin AV. Effect of two types of stress (heat shock/high temperature and malnutrition/serum deprivation) on porcine ovarian cell functions and their response to hormones. Exp Biol. 2010; 213: $377-$ 382 .
[11] Isom SC, Prather RS, Rucker EB. Heat stress-induced apoptosis in porcine in vitro fertilized and parthenogenetic preimplantation-stage embryos. Mol Reprod Dev. 2007; 74: 574-581. PMid:17034050. https://doi.org/10.1002/mrd. 20620

[12] Hosseini SM, Hajian M, Nasr-Esfahani MH. Effects of heat shock during the early stage of oocyte maturation on the meiotic progression, subsequent embryonic development and gene expression in ovine. Zygote. 2015; 23: 573-582. PMid:24964001. https: //doi.org/10.1017/S0967199414000203

[13] Yang-yang W, Xiao-jing L. Effects of HSP70 siRNA on collagen expression in the human keloid fibroblasts. Shandong Medical Journal. 2018; 58: 17-20.

[14] Zeng F, Tee C, Liu M, et al. The p53/HSP70 inhibitor, 2phenylethynesulfonamide, causes oxidative stress, unfolded protein response and apoptosis in rainbow trout cells. Aquat Toxicol. 2014; 146: 45-51. PMid:24270669. https://doi.org/10.1016/j.aq uatox. 2013.10.026

[15] Wiech M, Olszewski MB, Tracz-Gaszewska Z, et al. Molecular mechanism of mutant p53 stabilization: the role of HSP70 and MDM2. P1os One. 2012; 7: e51426. PMid:23251530. https: //doi.org/10.1371/journal.pone.0051426

[16] Mei-hua S, Wen-yan J, Yao Z, et al. Melatonin Ameliorates Glucose Metabolism via Inhibiting ROS Generation in Insulin Resistant HepG2 Cells Induced by High Glucose and Insulin. Journal of University of South China (Medical Edition). 2014; 42: 433-435.

[17] Liu S, Dai X. Cai L, et al. Effect of Hsp27 on early embryonic development in the mouse. Reprod Biomed Online. 2013; 26: 491-499. PMid:23419798. https://doi.org/10.1016/j.rbmo.2013.01 .005

[18] Ying F, Min T, Yang F, et al. Investigation of protection of melatonin to mitochondrial DNA in the oxidative stress model of retinal pigment epithelial cells in vitro. Recent Advances in Ophthalmology. 2006; 26: 911-915. 